\title{
A Note on the Generalized Camassa-Holm Equation
}

\author{
Yun Wu and Ping Zhao \\ Department of Mathematics and Computer Science, Guizhou Normal University, Guiyang, Guizhou 550001, China
}

Correspondence should be addressed to Yun Wu; yunwu@gznu.edu.cn

Received 15 October 2014; Accepted 24 November 2014; Published 25 December 2014

Academic Editor: Naseer Shahzad

Copyright (C) $2014 \mathrm{Y}$. Wu and P. Zhao. This is an open access article distributed under the Creative Commons Attribution License, which permits unrestricted use, distribution, and reproduction in any medium, provided the original work is properly cited.

We study the generalized Camassa-Holm equation which contains the Camassa-Holm $(\mathrm{CH})$ equation and Novikov equation as special cases with the periodic boundary condition. We get a blow-up scenario and obtain the global existence of strong and weak solutions under suitable assumptions, respectively. Then, we construct the periodic peaked solutions and apply them to prove the ill-posedness in $H^{s}$ with $s<3 / 2$.

\section{Introduction}

In this paper we study the global strong and weak solutions to the generalized Camassa-Holm equation with periodic boundary condition:

$$
\begin{gathered}
u_{t}-u_{x x t}+(n+2) u^{n} u_{x} \\
=(n+1) u^{n-1} u_{x} u_{x x}+u^{n} u_{x x x}, \quad t \geq 0, x \in \mathbb{T}, \\
u(0, x)=u_{0}(x), \quad x \in \mathbb{T},
\end{gathered}
$$

where $n \geq 1, n \in \mathbb{N}$, and $\mathbb{T}=\mathbb{R} / 2 \pi \mathbb{Z}$.

When $n=1$, (1) reduces to the the well-known $\mathrm{CH}$ equation:

$$
u_{t}-u_{x x t}+3 u u_{x}=2 u_{x} u_{x x}+u u_{x x x} \text {. }
$$

The $\mathrm{CH}$ equation was derived independently by Fokas and Fuchssteiner in [1] and by Camassa and Holm in [2]. Fokas and Fuchssteiner derived (3) in studying completely integrable generalizations of the $\mathrm{KdV}$ equation with biHamiltonian structures, while Camassa and Holm proposed (3) to describe the unidirectional propagation of shallow water waves over a flat bottom.

As shown in [2], the $\mathrm{CH}$ equation is completely integrable and possesses an infinite number of conservation laws. Moreover, the $\mathrm{CH}$ equation is such an equation that exhibits both phenomena of soliton interaction (peaked soliton solutions) and wave breaking (the solution remains bounded while its slope becomes unbounded in finite time [3]), while the
$\mathrm{KdV}$ equation does not model breaking waves [4]. In fact, wave breaking is one of the most intriguing long-standing problems of water wave theory [5]. The essential feature of $\mathrm{CH}$ should be pointed out: the fact that the traveling waves have a peak at their crest is exactly like for the waves of greatest height solutions of the governing equations for water waves (see [6-8] for the details).

From a mathematical point of view the Camassa-Holm equation is well studied and a series of achievements had been made. Constantin [9] and Misiołek [10] investigated the Cauchy problem for the periodic Camassa-Holm equation. Constantin et al. $[3,11-14]$ studied the wave breaking of the Cauchy problem for the $\mathrm{CH}$ equation. Recently, Jiang et al. gave a new and direct proof for McKean's theorem in [15]. Xin and Zhang [16] proved that (3) has global weak solutions for initial data in $H^{1}(\mathbb{R})$. Bressan and Constantin developed a new approach to the analysis of the $\mathrm{CH}$ equation and proved the existence of the global conservative and dissipative solutions in $[17,18]$. Holden and Raynaud $[19,20]$ also obtained the global conservative and dissipative solutions. The large time behavior of the $\mathrm{CH}$ equation was firstly established in [21]. In [22], Himonas et al. studied the persistence properties and infinite propagation speed for the $\mathrm{CH}$ equation.

In 2009, Novikov [23] found a new integrable equation:

$$
u_{t}-u_{x x t}+4 u^{2} u_{x}=3 u u_{x} u_{x x}+u^{2} u_{x x x} .
$$

It is derived that (4) possesses a bi-Hamiltonian structure and an infinite sequence of conserved quantities and admits exact peaked solutions $u(t, x)= \pm \sqrt{c} e^{-|x-c t|}$ with $c>0[24,25]$, as 
well as the explicit formulas for multipeakon solutions [25, 26].

By using the Littlewood-Paley decomposition and Kato's theory, the well-posedness of the Novikov equation has been studied in Besov spaces $B_{p, r}^{s}(\mathbb{R})$ and in the Sobolev space $H^{s}(\mathbb{R})$ (see $[27,28]$ ). Wu and Yin [29] established some results on the existence and uniqueness of global weak solutions to the Novikov equation. Jiang and Ni [30] established some results about blow-up phenomena of the strong solution to the Cauchy problem for (4). For the periodic boundary condition case, Tiglay [31] proved that for $s>5 / 2$ the periodic Novikov equation is locally well-posed in $H^{s}(\mathbb{T})$. Later the range of regularity index of local well-posedness was extended to $s>3 / 2$ in [32]; furthermore, it is shown that the solution maps for both periodic boundary value problem and Cauchy problem of the Novikov equation are not uniformly continuous from any bounded subset in $H^{s}$ into $C\left([0, T] ; H^{s}\right)$. When $s<3 / 2$, Grayshan [33] proved that the properties of the solution map for (4) are not (globally) uniformly continuous in Sobolev spaces $H^{s}$. For the nonuniform dependence and ill-posedness results in Besov spaces, we refer to [34-37].

In this paper we consider the generalized $\mathrm{CH}$ equation (1) with $n \geq 3$. Let $y=u-u_{x x}$, then (1) takes the form of a quasilinear evolution equation of hyperbolic type:

$$
y_{t}+(n+1) u^{n-1} u_{x} y+u^{n} y_{x}=0, \quad x \in \mathbb{T}, t \geq 0 \text {. }
$$

Applying the operator $\left(1-\partial_{x}^{2}\right)^{-1}$, (1) can be expressed as the following nonlocal form:

$$
\begin{aligned}
u_{t} & +u^{n} u_{x}+\left(1-\partial_{x}^{2}\right)^{-1} \partial_{x}\left(u^{n+1}+\frac{2 n-1}{2} u^{n-1} u_{x}^{2}\right) \\
& +\left(1-\partial_{x}^{2}\right)^{-1}\left(\frac{n-1}{2} u^{n-2} u_{x}^{3}\right)=0
\end{aligned}
$$

From the above equation, we can view (1) as a nonlocal perturbation of the Burgers-type equation:

$$
u_{t}+u^{n} \partial_{x} u=0
$$

We recall the following results in [38] for (1) and (2).

Proposition 1. If $s>3 / 2$ and $u_{0} \in H^{s}$, then there exists a $T=T\left(u_{0}\right)>0$ such that (1) and (2) have a unique solution $u \in$ $C\left([0, T] ; H^{s}\right) \bigcap C^{1}\left([0, T] ; H^{s-1}\right)$ which depends continuously on the initial data $u_{0}$. Moreover, $u$ satisfies the solution estimate

$$
\|u(t)\|_{H^{s}} \leq 2\left\|u_{0}\right\|_{H^{s}}, \quad 0 \leq t \leq T=\frac{1}{2 n c_{s}\left\|u_{0}\right\|_{H^{s}}^{n}}
$$

where $c_{s}>0$ is a constant depending on $s$.

In this paper we use the following notations. We use $\lesssim$ to denote estimates that hold up to some universal constant which may change from line to line but whose meaning is clear from the context. $f \approx g$ stands for $f \leqslant g$ and $f \geq g$. All function spaces are over $\mathbb{T}$ and we drop $\mathbb{T}$ in all function spaces if there is no ambiguity. For linear operators $A$ and $B$, we denote $[A, B]=A B-B A$. The Fourier transform of the function $f(x)$ is defined by $\widehat{f}(\xi)=\mathscr{F} f(\xi)=\int_{\mathbb{T}} e^{-i x \xi} f(x) d x$, $\xi \in \mathbb{Z}$. The inverse Fourier transform is given by $f(x)=$ $\mathscr{F}^{-1} \widehat{f}(x)=(1 / 2 \pi) \sum_{\xi \in \mathbb{Z}} \widehat{f}(\xi) e^{i x \xi}$.

The operator $D^{s}=\left(1-\partial_{x}^{2}\right)^{s / 2}$ for any real number $s$ is defined by $\widehat{D^{s} f}(\xi)=\left(1+\xi^{2}\right)^{s / 2} \widehat{f}(\xi) . H^{s}(\mathbb{T})$ is the standard Soblev space on $\mathbb{T}$ whose norm is defined by

$$
\|f\|_{H^{s}}^{2}=\sum_{\xi \in \mathbb{Z}}\left(1+\xi^{2}\right)^{s}|\widehat{f}|^{2}=\left\|D^{s} f\right\|_{L^{2}}^{2}
$$

For each $\varepsilon \in(0,1], J_{\varepsilon}$ stands for the Friedrichs mollifier defined by

$$
J_{\varepsilon} f(x)=j_{\varepsilon} * f(x),
$$

where $*$ stands for the convolution. Here $j_{\varepsilon}(x)=\mathscr{F}^{-1}(\widehat{j}(\varepsilon \xi))$ and $j(x)$ is a Schwartz function satisfying $0 \leq \widehat{j}(\xi) \leq 1$ for all the $\xi \in \mathbb{R}$ and $\hat{j}(\xi)=1$ for any $\xi \in[-1,1]$.

We will also use another mollifier. Define

$$
\rho(x)= \begin{cases}C \exp \left(\frac{1}{x^{2}-1}\right), & |x|<1, \\ 0, & |x| \geq 1\end{cases}
$$

where the constant $C>0$ is chosen so that $\int_{\mathbb{R}} \rho(x) d x=1$. For $\varepsilon \in(0,1]$, we set $\rho_{\varepsilon}(x)=(1 / \varepsilon) \rho((1 / \varepsilon) x)$. To define the mollifier $\widetilde{J}_{\varepsilon}$, we first let $\chi_{\Omega}$ be the characteristic function on $\Omega \subset \mathbb{R}$ and

$$
\tilde{f}_{\varepsilon}(x)=\rho_{\varepsilon / 2} *\left(\chi_{[\varepsilon, 2 \pi-\varepsilon]} f(x)\right), \quad x \in[0,2 \pi] .
$$

It follows that supp $\tilde{f}_{\varepsilon} \subset[\varepsilon / 2,2 \pi-\varepsilon / 2]$ and when $f$ is smooth, $\tilde{f}_{\varepsilon}-\chi_{[\varepsilon, 2 \pi-\varepsilon]} f \rightarrow 0$ as $\varepsilon \rightarrow 0$ and $x \in[0,2 \pi]$. Then we can define $\widetilde{J}_{\varepsilon}$ by

$\widetilde{J}_{\varepsilon} f=$ the unique periodic extension of $\widetilde{f}_{\varepsilon}$ with period $2 \pi$.

Obviously, if $f \in H^{s}(\mathbb{\mathbb { T }}), s>0$, then $\widetilde{J}_{\varepsilon} f \in H^{\infty}(\mathbb{\mathbb { T }})$ and $\| f-$ $\tilde{J}_{\varepsilon} f \|_{H^{s}} \rightarrow 0($ as $\varepsilon \rightarrow 0)$. Moreover, if $f(x) \geq 0$, then $\widetilde{J}_{\varepsilon} f \geq 0$. Now we present our results.

Theorem 2. Let $u_{0} \in H^{s}, s>3 / 2$, and let $T_{u_{0}}^{\star}$ be the maximal existence time of the corresponding solution $u$ to (1) and (2). Then $u$ blows up at $T_{u_{0}}^{\star}<\infty$ if and only if

$$
\limsup _{t \rightarrow T_{u_{0}}^{\star}}\left\|u_{x}(t)\right\|_{L^{\infty}}=\infty \text {. }
$$

Theorem 3. Let $u_{0} \in H^{s}, s>3 / 2$. If $u_{0}-\partial_{x}^{2} u_{0}$ does not change sign, then the corresponding solution to (1) and (2) exists globally.

Theorem 4. Suppose $u_{0} \in H^{s}$ with $s \in(1,3 / 2]$ such that $\left(1-\partial_{x}^{2}\right) u_{0}$ does not change sign. Then (1) and (2) have a unique global weak solution $u \in L_{\mathrm{loc}}^{\infty}\left([0, \infty) ; H^{s}\right)$ in the sense of distribution. Moreover, $u_{x} \in L_{\mathrm{loc}}^{\infty}([0, \infty) \times \mathbb{T})$.

When $s<3 / 2$, the solution map is not uniformly continuous, and we establish the ill-posedness as follows. We refer to $[33,36,39]$ for the ill-posedness results for the $\mathrm{CH}$ equation, Novikov equation, and the b-family equation. 
Theorem 5. If $s<3 / 2$, then (1) and (2) are ill-posed in $H^{s}$ in the sense that the solution map is not uniformly continuous from $H^{s}$ into $C\left([0, T] ; H^{s}\right)$ for any $T>0$. More precisely, there exist two sequences of weak solutions $u_{m}$ and $v_{m}$ in $H^{s}$ of (1) and (2) such that, for any $t \in[0, T]$, there hold the following estimates:

$$
\begin{gathered}
\left\|u_{m}(0, \cdot)-v_{m}(0, \cdot)\right\|_{H^{s}} \leq \frac{C_{1}(s)}{m}, \\
\left\|u_{m}(t, \cdot)-v_{m}(t, \cdot)\right\|_{H^{s}} \geq C_{2}(s) t^{3 / 2-s} m^{s+2|s|+1 / 2},
\end{gathered}
$$

where $C_{1}(s), C_{2}(s)$ only depend on $s$.

We outline the rest of the paper. In the next section, we give some preliminaries. We deal with the blow-up criterion and prove Theorems 2 and 3 in Section 3. In Section 4, we study the global weak solution and prove Theorem 4 . We demonstrate Theorem 5 in Section 5.

\section{Preliminaries}

Rewrite (1) and (2) as follows:

$$
\begin{gathered}
\partial_{t} u+u^{n} \partial_{x} u+F(u)=0, \quad x \in \mathbb{T}, t \in \mathbb{R}^{+}, \\
u(0, x)=u_{0}(x) \in H^{s}, \quad x \in \mathbb{T},
\end{gathered}
$$

where $F(u)=F_{1}(u)+F_{2}(u)+F_{3}(u)$, with

$$
\begin{aligned}
& F_{1}(u)=D^{-2} \partial_{x}\left(u^{n+1}\right), \\
& F_{2}(u)=\frac{2 n-1}{2} D^{-2} \partial_{x}\left[u^{n-1}\left(\partial_{x} u\right)^{2}\right], \\
& F_{3}(u)=\frac{n-1}{2} D^{-2}\left[u^{n-2}\left(\partial_{x} u\right)^{3}\right] .
\end{aligned}
$$

The following estimates are useful in our work.

Lemma 6 (Kato-Ponce commutator estimate [40]). If $s>0$, $f \in H^{s} \cap W^{1, \infty}$, and $g \in H^{s-1} \cap L^{\infty}$, then

$$
\begin{aligned}
& \left\|\left[D^{s}, f\right] g\right\|_{L^{2}} \\
& \quad \leq C_{s}\left(\left\|D^{s} f\right\|_{L^{2}}\|g\|_{L^{\infty}}+\left\|\partial_{x} f\right\|_{L^{\infty}}\left\|D^{s-1} g\right\|_{L^{2}}\right) .
\end{aligned}
$$

Lemma 7 (see [40] or see the Moser estimate in [41]). If $s>0$, then $H^{s} \cap L^{\infty}$ is an algebra, and

$$
\begin{array}{r}
\|f g\|_{H^{s}} \leq C_{s}\left(\|f\|_{H^{s}}\|g\|_{L^{\infty}}+\|f\|_{L^{\infty}}\|g\|_{H^{s}}\right), \\
\forall f, g \in H^{s} \bigcap L^{\infty} .
\end{array}
$$

From the construction of the mollifier $j_{\varepsilon}$, we know that $\widehat{j}_{\mathcal{\varepsilon}}(\xi)=\widehat{j}(\varepsilon \xi)$ and, for any $u \in H^{s}$ and $r \leq s$,

$$
\left\|u-J_{\varepsilon} u\right\|_{H^{r}}=o\left(\varepsilon^{s-r}\right) .
$$

In addition, we have

$$
\begin{aligned}
D^{s} J_{\varepsilon} & =J_{\varepsilon} D^{s}, \\
\left(J_{\varepsilon} f, g\right)_{L^{2}} & =\left(f, J_{\varepsilon} g\right)_{L^{2}}, \\
\left\|J_{\varepsilon} u\right\|_{H^{s}} & \leq\|u\|_{H^{s}} .
\end{aligned}
$$

Lemma 8 (see [42]). Let $w(x)$ be a function such that $\left\|\partial_{x} w\right\|_{L^{\infty}}<\infty$. Then, there is a $c>0$ such that, for any $f \in L^{2}$,

$$
\left\|\left[J_{\varepsilon}, w\right] \partial_{x} f\right\|_{L^{2}} \leq c\left\|\partial_{x} w\right\|_{L^{\infty}}\|f\|_{L^{2}} .
$$

The following Calderon-Coifman-Meyer type commutator estimate is also useful (see Proposition 4.2, [43]).

Lemma 9. If $\rho>3 / 2$ and $0 \leq \sigma+1 \leq \rho$, then there is a $c>0$ such that

$$
\left\|\left[D^{\sigma} \partial_{x}, f\right] v\right\|_{L^{2}} \leq c\|f\|_{H^{\rho}}\|v\|_{H^{\sigma}}, \quad \forall f \in H^{\rho}, \quad v \in H^{\sigma} .
$$

\section{Blow-Up Criterion and Global Existence of Strong Solutions}

The aim of this section is to prove Theorems 2 and 3 which show that the solution blows up only when the slope of the wave blows up and the solution exists globally if $y_{0}=u_{0}-u_{0 \times x}$ does not change sign. Rewrite (1) as the following form:

$$
\begin{aligned}
u_{t}-u_{x x t}= & -(n+2)\left(u^{n} u_{x}\right)+\partial_{x}^{2}\left(u^{n} u_{x}\right) \\
& -n \partial_{x}\left(u^{n-1} u_{x}^{2}\right)+u^{n-1} u_{x} u_{x x} .
\end{aligned}
$$

First, we have the following lemma.

Lemma 10. Let $s>3 / 2,0<q \leq s-1$, and $u(t, x)$ is a solution to (1) and (2) with $u_{0} \in H^{s}$. Then there exists a constant $C_{s}$ such that

$$
\begin{aligned}
& \|u(t)\|_{H^{q+1}}^{2}-\|u(0)\|_{H^{q+1}}^{2} \\
& \quad \leq C_{s} \int_{0}^{t}\|u\|_{H^{q+1}}^{2}\left(\|u\|_{L^{\infty}}^{n-1}\left\|u_{x}\right\|_{L^{\infty}}+\|u\|_{L^{\infty}}^{n-2}\left\|u_{x}\right\|_{L^{\infty}}^{2}\right) d \tau .
\end{aligned}
$$

Proof. Since the term $-n \partial_{x}\left(u^{n-1} u_{x}^{2}\right)+u^{n-1} u_{x} u_{x x}$ is only in $H^{s-2}$, we cannot apply $D^{q}$ to either side of (26) when $q>s-2$. So we apply the operator $D^{q} J_{\varepsilon}$ to (26), multiply both sides of the resulting equation by $2 D^{q} J_{\varepsilon} u$, and integrate over $\mathbb{T}$ to obtain

$$
\begin{aligned}
\frac{d}{d t} \int_{\mathbb{T}} & {\left[\left(D^{q} J_{\varepsilon} u\right)^{2}+\left(D^{q} J_{\varepsilon} u_{x}\right)^{2}\right] d x } \\
= & -2(n+1) \int_{\mathbb{T}} D^{q} J_{\varepsilon} u \cdot D^{q} J_{\varepsilon}\left(u^{n} u_{x}\right) d x \\
& -2 \int_{\mathbb{T}} D^{q+1} J_{\varepsilon} u \cdot D^{q+1} J_{\varepsilon}\left(u^{n} u_{x}\right) d x \\
& +2 n \int_{\mathbb{T}} D^{q} J_{\varepsilon} u_{x} \cdot D^{q} J_{\varepsilon}\left(u^{n-1} u_{x}^{2}\right) d x \\
& +2 \int_{\mathbb{T}} D^{q} J_{\varepsilon} u \cdot D^{q} J_{\varepsilon}\left(u^{n-1} u_{x} u_{x x}\right) d x \\
= & N_{1}+N_{2}+N_{3}+N_{4},
\end{aligned}
$$


where we used $\partial_{x}^{2}=1-D^{2}$ and

$$
\begin{aligned}
\int_{\mathbb{T}} & D^{q} J_{\varepsilon} u \cdot D^{q} \partial_{x}^{2} J_{\varepsilon}\left(u^{n} u_{x}\right) d x \\
= & \int_{\mathbb{T}} D^{q} J_{\varepsilon} u \cdot D^{q} J_{\varepsilon}\left(u^{n} u_{x}\right) d x \\
& \quad-\int_{\mathbb{T}} D^{q+1} J_{\varepsilon} u \cdot D^{q+1} J_{\varepsilon}\left(u^{n} u_{x}\right) d x .
\end{aligned}
$$

We now estimate $N_{i}, 1 \leq i \leq 4$, respectively. For $N_{1}$, we first note that $J_{\varepsilon}$ is self-adjoint, then commute the operator $D^{q}$ with $u^{n}$, and use (21) and (22) to get

$$
\begin{aligned}
N_{1}= & \int_{\mathbb{T}}\left[D^{q}, u^{n}\right] \partial_{x} u D^{q} J_{\varepsilon}^{2} u d x \\
& +\int_{\mathbb{T}}\left[J_{\varepsilon}, u^{n}\right] \partial_{x} D^{q} u D^{q} J_{\varepsilon} u d x \\
& -\frac{1}{2} \int_{\mathbb{T}}\left(u^{n}\right)_{x}\left(D^{q} J_{\varepsilon} u\right)^{2} d x .
\end{aligned}
$$

By using the Cauchy-Schwarz inequality, Lemmas 6, 7, and 8 , integration by parts, and (23), we have

$$
\begin{aligned}
& \left|\int_{\mathbb{T}}\left[D^{q}, u^{n}\right] \partial_{x} u D^{q} J_{\varepsilon}^{2} u d x\right| \\
& \quad \leq\left(\left\|u^{n}\right\|_{H^{q}}\left\|\partial_{x} u\right\|_{L^{\infty}}+\left\|\partial_{x}\left(u^{n}\right)\right\|_{L^{\infty}}\left\|\partial_{x} u\right\|_{H^{q-1}}\right)\|u\|_{H^{q}} \\
& \quad \leq\|u\|_{L^{\infty}}^{n-1}\left\|u_{x}\right\|_{L^{\infty}}\|u\|_{H^{q}}^{2}, \\
& \quad \leq \| \int_{\mathbb{T}}\left[J_{\varepsilon}, u^{n}\right] \partial_{x} D^{q} u D^{q} J_{\varepsilon} u d x \mid \\
& \quad \leq\left\|\left[J_{\varepsilon}, u^{n}\right] D^{q} \partial_{x} u\right\|_{L^{2}}^{n-1}\|u\|_{H^{q}} \\
& \quad \frac{1}{2} \int_{\mathbb{T}} \partial_{x}\left(u^{n}\right)\left(u_{x}\left\|_{L^{\infty}}\right\| u \|_{H^{q}}^{2},\right. \\
& \quad \leq\left\|\left(u^{n}\right)_{x}\right\|_{L^{\infty}} \|^{2} d x \mid
\end{aligned}
$$

Therefore

$$
\left|N_{1}\right| \lesssim\|u\|_{L^{\infty}}^{n-1}\left\|u_{x}\right\|_{L^{\infty}}\|u\|_{H^{q}}^{2} .
$$

In the same way, $N_{2}$ can be estimated as

$$
\left|N_{2}\right| \lesssim\|u\|_{L^{\infty}}^{n-1}\left\|u_{x}\right\|_{L^{\infty}}\|u\|_{H^{q+1}}^{2} .
$$

For $\mathrm{N}_{3}$, we use the Cauchy-Schwarz inequality, Lemma 7, and (23) to obtain

$$
\begin{aligned}
& \left|N_{3}\right| \leq\|u\|_{H^{q+1}}\left\|\partial_{x}\left(u^{n}\right) u_{x}\right\|_{H^{q}} \\
& \leq\|u\|_{H^{q+1}}\left(\left\|\partial_{x}\left(u^{n}\right)\right\|_{H^{q}}\left\|u_{x}\right\|_{L^{\infty}}\right. \\
& \left.\quad+\|u\|_{L^{\infty}}^{n-1}\left\|u_{x}\right\|_{L^{\infty}}\|u\|_{H^{q+1}}\right) \\
& \leq\|u\|_{L^{\infty}}^{n-1}\left\|u_{x}\right\|_{L^{\infty}}\|u\|_{H^{q+1}}^{2} .
\end{aligned}
$$

For the estimate for $\mathrm{N}_{4}$, we have

$$
\begin{aligned}
\left|N_{4}\right|=2 \mid & \int_{\mathbb{T}} D^{q} J_{\varepsilon} u \cdot D^{q} J_{\varepsilon} \\
& \times\left[\partial_{x}\left(u^{n-1} u_{x}^{2}\right)-(n-1) u^{n-2} u_{x}^{3}\right] d x \mid \\
\lesssim & \left|\int_{\mathbb{T}} D^{q} J_{\varepsilon} u_{x} \cdot D^{q} J_{\varepsilon}\left(u^{n-1} u_{x}^{2}\right) d x\right| \\
& +\left|\int_{\mathbb{T}} D^{q} J_{\varepsilon} u \cdot D^{q} J_{\varepsilon}\left(u^{n-2} u_{x}^{3}\right) d x\right| \\
= & K_{1}+K_{2} .
\end{aligned}
$$

Similar to (34), $K_{1} \leq\|u\|_{L^{\infty}}^{n-1}\left\|u_{x}\right\|_{L^{\infty}}\|u\|_{H^{q+1}}^{2}$, while

$$
\begin{aligned}
K_{2} & \leq\|u\|_{H^{q}}\left(\left\|u^{n-2} u_{x}\right\|_{L^{\infty}}\left\|u_{x}^{2}\right\|_{H^{q}}+\left\|u^{n-2} u_{x}\right\|_{H^{q}}\left\|u_{x}\right\|_{L^{\infty}}^{2}\right) \\
& \leq\|u\|_{H^{q}}\left(\|u\|_{L^{\infty}}^{n-2}\left\|u_{x}\right\|_{L^{\infty}}^{2}\left\|u_{x}\right\|_{H^{q}}+\left\|\partial_{x}\left(u^{n-1}\right)\right\|_{H^{q}}\left\|u_{x}\right\|_{L^{\infty}}^{2}\right) \\
& \leq\|u\|_{H^{q}}\left(\|u\|_{L^{\infty}}^{n-2}\left\|u_{x}\right\|_{L^{\infty}}^{2}\left\|u_{x}\right\|_{H^{q}}+\|u\|_{H^{q+1}}\|u\|_{L^{\infty}}^{n-2}\left\|u_{x}\right\|_{L^{\infty}}^{2}\right) \\
& \leq\|u\|_{L^{\infty}}^{n-2}\left\|u_{x}\right\|_{L^{\infty}}^{2}\|u\|_{H^{q+1}}^{2} .
\end{aligned}
$$

Hence we obtain that

$$
\left|N_{4}\right| \lesssim\|u\|_{L^{\infty}}^{n-2}\left\|u_{x}\right\|_{L^{\infty}}^{2}\|u\|_{H^{q+1}}^{2} .
$$

Combining (32), (33), (34), and (37) yields

$$
\begin{aligned}
& \frac{d}{d t} \int_{\mathbb{T}}\left[\left(D^{q} J_{\varepsilon} u\right)^{2}+\left(D^{q} J_{\varepsilon} u_{x}\right)^{2}\right] d x \\
& \quad \leq\|u\|_{H^{q+1}}^{2}\left(\|u\|_{L^{\infty}}^{n-1}\left\|u_{x}\right\|_{L^{\infty}}+\|u\|_{L^{\infty}}^{n-2}\left\|u_{x}\right\|_{L^{\infty}}^{2}\right) .
\end{aligned}
$$

Integrating both sides with respect to $t$ results in

$$
\begin{aligned}
& \left\|J_{\varepsilon} u(t)\right\|_{H^{q+1}}^{2}-\left\|J_{\mathcal{\varepsilon}} u(0)\right\|_{H^{q+1}}^{2} \\
& \quad \leq \int_{0}^{t}\|u\|_{H^{q+1}}^{2}\left(\|u\|_{L^{\infty}}^{n-1}\left\|u_{x}\right\|_{L^{\infty}}+\|u\|_{L^{\infty}}^{n-2}\left\|u_{x}\right\|_{L^{\infty}}^{2}\right) d \tau .
\end{aligned}
$$

Let $\varepsilon$ tend to 0 ; we get (27) and therefore complete the proof of this lemma.

Remark 11. Take $q^{\prime}=q+1$ in (27); then for $q^{\prime} \in(1, s]$, we have

$$
\begin{aligned}
& \|u(t)\|_{H^{q^{\prime}}}^{2}-\|u(0)\|_{H^{q^{\prime}}}^{2} \\
& \left.\quad \leq C_{s} \int_{0}^{t}\|u\|_{H^{q^{\prime}}}^{2}\|u\|_{L^{\infty}}^{n-1}\left\|u_{x}\right\|_{L^{\infty}}+\|u\|_{L^{\infty}}^{n-2}\left\|u_{x}\right\|_{L^{\infty}}^{2}\right) d \tau .
\end{aligned}
$$

This estimate will be also used in the proofs of Lemma 20.

Let $u$ be the solution to problem (1) and (2). We define $T_{u_{0}}^{*}$ to be its lifespan,

$$
T_{u_{0}}^{\star}=\sup \left\{T>0: u \in C\left([0, T) ; H^{s}\right) \bigcap C^{1}\left([0, T) ; H^{s-1}\right)\right\} .
$$


Then the following alternative property holds:

$$
\begin{aligned}
& \text { (i) } T_{u_{0}}^{\star}=\infty, \quad \text { or } \\
& \text { (ii) } T_{u_{0}}^{\star}<\infty, \quad \lim _{t \rightarrow T_{u_{0}}^{\star}}\|u(t, \cdot)\|_{H^{s}}=\infty \text {. }
\end{aligned}
$$

Proof of Theorem 2. From (1) we can deduce that

$$
\frac{d}{d t} \int_{\mathbb{T}}\left(u^{2}+u_{x}^{2}\right) d x=0
$$

which implies that

$$
\|u\|_{H^{1}}=\left\|u_{0}\right\|_{H^{1}}
$$

Taking $q=s-1$ in Lemma 10 and using $\|u\|_{L^{\infty}} \lesssim\|u\|_{H^{1}}=$ $\left\|u_{0}\right\|_{H^{1}} \lesssim 1$, we obtain that

$$
\|u\|_{H^{s}} \leq\left\|u_{0}\right\|_{H^{s}} \exp \left(C_{s} \int_{0}^{t}\left(\left\|u_{x}\right\|_{L^{\infty}}+\left\|u_{x}\right\|_{L^{\infty}}^{2}\right) d \tau\right) .
$$

Hence we know that if $\lim \sup _{t \rightarrow T_{u_{0}}^{\star}}\left\|u_{x}(t)\right\|_{L^{\infty}}$ is finite, then $\|u(t)\|_{H^{s}}$ is bounded and the case (ii) in (42) would not occur, which implies that $u$ can be extended beyond $T_{u_{0}}^{\star}$. On the other hand, if $u$ does not blow up, then $\|u(t)\|_{H^{s}}$ is bounded on $[0, T]$ for any $T>0$. Since $H^{s-1} \hookrightarrow L^{\infty},\left\|u_{x}\right\|_{L^{\infty}}$ is also bounded on $\left[0, T_{u_{0}}^{\star}\right]$. Thus we complete the proof.

Remark 12. Actually, we can prove a more precise blow-up criterion for sufficiently regular solutions to (1); that is, if $u_{0} \in$ $H^{s}$, with $s \geq 2$, then the solution $u(t, x)$ blows up if and only if

$$
\liminf _{t \rightarrow T_{u_{0}}^{\star}}\left\{\inf _{x \in \mathbb{T}}\left[u^{n-1} u_{x}\right](x, t)\right\}=-\infty
$$

In fact, multiplying both sides of (5) by $y=u-u_{x x}$ and integrating over $\mathbb{T}$, we have

$$
\begin{aligned}
& \frac{1}{2} \frac{d}{d t} \int_{\mathbb{T}} y^{2} d x \\
& \quad=-(n+1) \int_{\mathbb{T}} u^{n-1} u_{x} y^{2} d x-\int_{\mathbb{T}} u^{n} y_{x} y d x \\
& \quad=\left(-\frac{n+2}{2}\right) \int_{\mathbb{T}} u^{n-1} u_{x} y^{2} d x .
\end{aligned}
$$

If $u^{n-1} u_{x}$ is bounded from below on $\left[0, T_{u_{0}^{*}}^{*}\right) \times \mathbb{T}$, that is, for some $M>0, u^{n-1} u_{x}>-M$ for $(t, x) \in\left[0, T_{u_{0}}^{\star}\right) \times \mathbb{T}$, then

$$
\int_{\mathbb{T}} y^{2} d x \leq \frac{(n+2) M}{2} \int_{0}^{t} \int_{\mathbb{T}} y^{2} d x+C,
$$

where $C>0$. By Gronwall's inequality, we obtain that the $L^{2}$ norm of $y$ is bounded on $\left[0, T_{u_{0}}^{\star}\right)$ which is equivalent to the boundedness of $\|u\|_{H^{2}}$ since $\|u\|_{H^{2}} \leq\|y\|_{L^{2}} \leq 2\|u\|_{H^{2}}$. On the other hand, since $s \geq 2$ and $H^{s-1}$ is an algebra, we know that $\left\|u^{n-1} u_{x}\right\|_{L^{\infty}} \lesssim\left\|u^{n-1}\right\|_{H^{s}}\left\|u_{x}\right\|_{H^{s-1}} \lesssim\|u\|_{H^{s}}^{n}$.
Remark 13. The new blow-up criterion (46) is better than the one obtained in Theorem 2, which is quite common for nonlinear hyperbolic PDE (see $[5,44]$ ). For the CamassaHolm and related equations, the blow-up criterion is often written as

$$
\liminf _{t \rightarrow T_{u_{0}}^{\star}}\left\{\inf _{x \in \mathbb{T}} u_{x}(t, x)\right\}=-\infty
$$

which is different from (46). In fact, if $u$ blows up and $u^{n-1}<$ 0 , then $u_{x} \rightarrow+\infty$.

Let $q(t, x)$ be the particle curve evolved by the solution; that is, it satisfies

$$
\begin{gathered}
\frac{d q(t, x)}{d t}=u^{n}(t, q(t, x)), \quad t \in\left[0, T_{u_{0}}^{\star}\right), \\
q(0, x)=x, \quad x \in \mathbb{R} .
\end{gathered}
$$

Since $u \in C\left(\left[0, T_{u_{0}}^{\star}\right) ; H^{s}\right) \bigcap C^{1}\left(\left[0, T_{u_{0}}^{\star}\right) ; H^{s-1}\right), s>3 / 2$, we see that $u(t, x)$ and $u_{x}(t, x)$ belong to $C^{1}([0, T] \times \mathbb{\mathbb { T }})$ for any $T<T_{u_{0}}^{\star}$. Therefore, for a fixed $x,(50)$ has a unique solution $q(t, x) \in C^{1}([0, T] \times \mathbb{R})$. Moreover, we have the following lemma.

Lemma 14. Let $u_{0} \in H^{s}, s>3 / 2$. The map $q(t, \cdot)$ is an increasing diffeomorphism of $\mathbb{R}$, and $q_{x}(t, x)>0$ for $(t, x) \in$ $\left[0, T_{u_{0}}^{\star}\right) \times \mathbb{R}$.

Proof. Differentiating (50) with respect to $x$ yields that

$$
\begin{gathered}
\frac{d q_{x}(t, x)}{d t}=n u^{n-1} u_{x}(t, q) q_{x}, \quad t \in\left[0, T_{u_{0}}^{\star}\right), \\
q_{x}(0, x)=1, \quad x \in \mathbb{R} .
\end{gathered}
$$

Solving the above equation, we obtain

$$
q_{x}(t, x)=\exp \left(n \int_{0}^{t} u^{n-1} u_{x}(\tau, q(\tau, x)) d \tau\right) .
$$

Thus $q_{x}$ is positive and $q(t, x)$ is an increasing diffeomorphism of $\mathbb{R}$ before the blow-up time.

The following property for the strong solution is important in the proof of global existence.

Lemma 15. Let $u_{0} \in H^{s}, s \geq 3$, and $u$ is the solution to (1) and (2). One has the following identity:

$$
y(t, q(t, x)) q_{x}^{(n+1) / n}(t, x)=y_{0}(x),
$$

where $(t, x) \in\left[0, T_{u_{0}}^{\star}\right) \times \mathbb{R}, y=u-u_{x x}$, and $y_{0}=u_{0}-u_{0 x x}$. Moreover, if $\left|y_{0}\right|^{n /(n+1)} \in L^{1}$, then

$$
\int_{\mathbb{T}}|y|^{n /(n+1)} d x=\int_{\mathbb{T}}\left|y_{0}\right|^{n /(n+1)} d x .
$$


Proof. Differentiating $y(t, q(t, x)) q_{x}^{(n+1) / n}(t, x)$ with respect to $t$, we have

$$
\begin{aligned}
\frac{d}{d t} & y(t, q(t, x)) q_{x}^{(n+1) / n}(t, x) \\
& =y_{t} q_{x}^{(n+1) / n}+\frac{n+1}{n} y q_{x}^{((n+1) / n)-1} q_{x t}+y_{x} q_{t} q_{x}^{(n+1) / n} \\
& =q_{x}^{(n+1) / n}\left(y_{t}+(n+1) y u^{n-1} u_{x}+y_{x} u^{n}\right)=0
\end{aligned}
$$

Solving the above equation with $q_{x}(0, x)=1$, we obtain (53). Furthermore, under the condition in the lemma, we have

$$
\begin{aligned}
\int_{\mathbb{T}}|y(t, x)|^{n /(n+1)} d x \\
=\int_{\mathbb{T}}|y(t, q(t, x))|^{n /(n+1)} q_{x}(t, x) d x \\
=\frac{d}{d t} \int_{\mathbb{T}}\left|y(t, q(t, x)) q_{x}^{(n+1) / n}(t, x)\right|^{n /(n+1)} d x \\
=\int_{\mathbb{T}}\left|y_{0}\right|^{n /(n+1)} d x,
\end{aligned}
$$

which implies (54).

Remark 16. If $u_{0}(x)$ has a compact support in an interval $[a, b]$, so does $y_{0}(x)=\left(1-\partial_{x}^{2}\right) u_{0}(x)$. Because of (53), we know $y(t, x)$ is compactly supported in $[q(t, a), q(t, b)]$ within its lifespan.

We note that the Green's function of $\left(1-\partial_{x}^{2}\right)^{-1}$ is $G_{\mathbb{T}}=$ $\cosh (x-2 \pi[x / 2 \pi]-\pi) / 2 \sinh (\pi)$ for $x \in \mathbb{T}$, where $[x]$ stands for the integer part of $x$. For $f \in L^{2}(\mathbb{T})$, we have

$$
\begin{aligned}
{\left[\left(1-\partial_{x}^{2}\right)^{-1} f\right](x) } & =G_{\mathbb{T}} * f(x) \\
& =\int_{\mathbb{T}} G_{\mathbb{T}}(x-\eta) f(\eta) d \eta .
\end{aligned}
$$

A direct computation gives rise to

$$
\begin{aligned}
u(t, x)= & \frac{e^{x-\pi}}{4 \sinh (\pi)} \int_{0}^{x} e^{-\xi} y(t, \xi) d \xi \\
& +\frac{e^{-x+\pi}}{4 \sinh (\pi)} \int_{0}^{x} e^{\xi} y(t, \xi) d \xi \\
& +\frac{e^{x+\pi}}{4 \sinh (\pi)} \int_{x}^{2 \pi} e^{-\xi} y(t, \xi) d \xi \\
& +\frac{e^{-x-\pi}}{4 \sinh (\pi)} \int_{x}^{2 \pi} e^{\xi} y(t, \xi) d \xi, \\
u_{x}(t, x)= & \frac{e^{x-\pi}}{4 \sinh (\pi)} \int_{0}^{x} e^{-\xi} y(t, \xi) d \xi \\
& -\frac{e^{-x+\pi}}{4 \sinh (\pi)} \int_{0}^{x} e^{\xi} y(t, \xi) d \xi \\
& +\frac{e^{x+\pi}}{4 \sinh (\pi)} \int_{x}^{2 \pi} e^{-\xi} y(t, \xi) d \xi \\
& -\frac{e^{-x-\pi}}{4 \sinh (\pi)} \int_{x}^{2 \pi} e^{\xi} y(t, \xi) d \xi .
\end{aligned}
$$

Therefore, we have

$$
\begin{aligned}
& {\left[u+u_{x}\right](t, x)} \\
& \quad=\frac{1}{2 \sinh (\pi)} \int_{0}^{2 \pi} e^{(x-\xi-2 \pi[(x-\xi) / 2 \pi]-\pi)} y(t, \xi) d \xi, \\
& {\left[u-u_{x}\right](t, x)} \\
& \quad=\frac{1}{2 \sinh (\pi)} \int_{0}^{2 \pi} e^{(-x+\xi+2 \pi[(x-\xi) / 2 \pi]+\pi)} y(t, \xi) d \xi .
\end{aligned}
$$

Lemma 17. If $u_{0} \in H^{s}, s>3 / 2$, and $y_{0}=\left(1-\partial_{x}^{2}\right) u_{0}(x)$ does not change sign, then the solution $u$ to (1) and (2) satisfies

$$
\left\|u_{x}\right\|_{L^{\infty}} \leq\|u\|_{L^{\infty}} \lesssim\|u\|_{H^{1}}=\left\|u_{0}\right\|_{H^{1}}, \quad x \in \mathbb{T}, t \in\left[0, T_{u_{0}}^{\star}\right) .
$$

Proof. We discuss the following results for the case $s \geq 3$; the lemma follows by using a simple density argument. By (53) and the positivity of $G_{\mathbb{T}}$, we know $y$ keeps the sign of $y_{0}$, and hence $u=G_{\mathbb{T}} * y$ keeps the sign of $y_{0}$. Therefore, employing (59), we obtain that, for $x \in \mathbb{T}, t \in\left[0, T_{u_{0}}^{\star}\right)$,

$$
\begin{aligned}
& -u \leq u_{x} \leq u, \quad \text { if } y_{0}=\left(1-\partial_{x}^{2}\right) u_{0}(x)>0, \quad x \in \mathbb{T}, \\
& u \leq u_{x} \leq-u, \quad \text { if } y_{0}=\left(1-\partial_{x}^{2}\right) u_{0}(x)<0, \quad x \in \mathbb{T} .
\end{aligned}
$$

That is, if $y_{0}$ does not change sign, then $\left|u_{x}\right| \leq|u|$. By $H^{1} \hookrightarrow$ $L^{\infty}$, we obtain the desired estimate.

Proof of Theorem 3. Combining Lemma 17 and Theorem 2, we have Theorem 3.

\section{Global Weak Solutions}

In this section, we prove that (1) and (2) have a unique global weak solution in lower-order Sobolev space $H^{s}, 1<s \leq 3 / 2$. First, we establish some estimates for the strong solutions to (1) with $s>3 / 2$.

Lemma 18. Let $s>3 / 2,0<q \leq s-1$, and $u \in C\left(\left[0, T_{u_{0}}^{\star}\right) ; H^{s}\right)$ is the solution to (1) and (2) with initial value $u_{0} \in H^{s}$. Then there is a constant $C_{s}$ such that, for $t \in\left[0, T_{u_{0}}^{\star}\right)$, one has

$$
\begin{aligned}
\left\|\partial_{t} u\right\|_{H^{q}} \leq & C_{s}\|u\|_{H^{q+1}} \\
& \times\left(\|u\|_{L^{\infty}}^{n}+\|u\|_{L^{\infty}}^{n-1}\left\|u_{x}\right\|_{L^{\infty}}+\|u\|_{L^{\infty}}^{n-2}\left\|u_{x}\right\|_{L^{\infty}}^{2}\right) .
\end{aligned}
$$

Proof. By using the operator $D^{-2}=\left(1-\partial_{x}^{2}\right)^{-1}$, we can rewrite (26) as

$$
\begin{aligned}
u_{t}= & D^{-2} \partial_{x}\left(-\frac{n+2}{n+1}\left(u^{n+1}\right)-n\left(u^{n-1} u_{x}^{2}\right)\right) \\
& +D^{-2} \partial_{x}^{2}\left(u^{n} u_{x}\right)+D^{-2}\left(u^{n-1} u_{x} u_{x x}\right) .
\end{aligned}
$$


For $q \in(0, s-1]$, taking the $H^{q}$ norm of both sides of (63), it follows that

$$
\begin{aligned}
\left\|u_{t}\right\|_{H^{q}} & \\
\leq & \left\|D^{-2} \partial_{x}\left(-\frac{n+2}{n+1}\left(u^{n+1}\right)-n\left(u^{n-1} u_{x}^{2}\right)\right)\right\|_{H^{q}} \\
& +\left\|D^{-2} \partial_{x}^{2}\left(u^{n} u_{x}\right)\right\|_{H^{q}}+\left\|D^{-2}\left(u^{n-1} u_{x} u_{x x}\right)\right\|_{H^{q}} \\
= & N_{1}^{\prime}+N_{2}^{\prime}+N_{3}^{\prime} .
\end{aligned}
$$

The estimate for $N_{i}^{\prime}(i=1,2,3)$ is straightforward. By using Lemma 7, we have

$$
\begin{aligned}
N_{1}^{\prime} & \lesssim\left\|u^{n+1}\right\|_{H^{q-1}}+\left\|\partial_{x}\left(u^{n}\right) u_{x}\right\|_{H^{q-1}} \\
& \lesssim\left\|u^{n+1}\right\|_{H^{q}}+\left\|\partial_{x}\left(u^{n}\right) u_{x}\right\|_{H^{q}} \\
& \lesssim\|u\|_{L^{\infty}}^{n}\|u\|_{H^{q+1}}+\|u\|_{L^{\infty}}^{n-1}\left\|u_{x}\right\|_{L^{\infty}}\|u\|_{H^{q+1}} .
\end{aligned}
$$

Using $\partial_{x}^{2}=1-D^{2}$, we have

$$
\begin{aligned}
\left|N_{2}^{\prime}\right| & \lesssim\left\|\partial_{x}\left(u^{n+1}\right)\right\|_{H^{q-2}}+\left\|\partial_{x}\left(u^{n+1}\right)\right\|_{H^{q}} \\
& \lesssim\|u\|_{L^{\infty}}^{n}\|u\|_{H^{q+1}} .
\end{aligned}
$$

Using Lemma 7, it follows that

$$
\begin{aligned}
\left|N_{3}^{\prime}\right| & \lesssim\left\|u^{n-1} \partial_{x}\left(u_{x}^{2}\right)\right\|_{H^{q-2}} \\
& \lesssim\left\|\partial_{x}\left(u^{n-1} u_{x}^{2}\right)-u_{x}^{2} \partial_{x}\left(u^{n-1}\right)\right\|_{H^{q-2}} \\
& \lesssim\left\|u^{n-1} u_{x}^{2}\right\|_{H^{q}}+\left\|u_{x}^{2} \partial_{x}\left(u^{n-1}\right)\right\|_{H^{q}} \\
& \lesssim\|u\|_{H^{q+1}}\left(\|u\|_{L^{\infty}}^{n-1}\left\|u_{x}\right\|_{L^{\infty}}+\|u\|_{L^{\infty}}^{n-2}\left\|u_{x}\right\|_{L^{\infty}}^{2}\right) .
\end{aligned}
$$

Inserting (65), (66), and (67) into (64) yields the inequality (62).

To show the existence of weak solution to (1) and (2) in lower-order Sobolev space $H^{s}$ with $1<s \leq 3 / 2$, we will consider the following problem first:

$$
\begin{gathered}
\partial_{t} u+u^{n} \partial_{x} u+F(u)=0, \quad x \in \mathbb{T}, t \in \mathbb{R}^{+}, \\
u(0, x)=\widetilde{J}_{\varepsilon} u_{0}(x) \in H^{\infty},
\end{gathered}
$$

where $F(u)$ is given in (17) and $\widetilde{J}_{\varepsilon}$ is the mollifier introduced in (11)-(13). It follows from Proposition 1 that, for each $\varepsilon>0$, there exists a $T_{\varepsilon}>0$ such that the above problem has a unique solution $u_{\varepsilon}(t, x) \in C^{\infty}\left(\left[0, T_{\varepsilon}\right] ; H^{\infty}\right)$.

Lemma 19. Let $u_{0} \in H^{s}, 1<s \leq 3 / 2$. If $\left(1-\partial_{x}^{2}\right) u_{0}$ does not change sign, then $u_{\varepsilon}(t, x) \in C\left([0, \infty) ; H^{\infty}\right)$ and $\left\|\partial_{x} u_{\varepsilon}\right\|_{L^{\infty}} \leq$ $\left\|u_{\varepsilon}\right\|_{L^{\infty}} \lesssim\left\|u_{0}\right\|_{H^{1}}$.

Proof. We first note that, by the construction of $\widetilde{J}_{\varepsilon}$, if $f \geq 0$ (or $f \leq 0$ ), then $\widetilde{J}_{\varepsilon} f \geq 0$ (or $\widetilde{J}_{\varepsilon} f \leq 0$ ). Thus, if $\left(1-\partial_{x}^{2}\right) u_{0}$ does not change sign, so does $\left(1-\partial_{x}^{2}\right) \widetilde{J}_{\varepsilon} u_{0}=\widetilde{J}_{\varepsilon}\left(1-\partial_{x}^{2}\right) u_{0}$. Using the notation $u_{\varepsilon}=u$ in (61) implies

$$
\left\|\partial_{x} u_{\varepsilon}\right\|_{L^{\infty}} \leq\left\|u_{\varepsilon}\right\|_{L^{\infty}} \lesssim\left\|u_{0}\right\|_{H^{1}} .
$$

Hence Theorem 3 yields that $u_{\varepsilon}(t, x)$ is a global solution.

Lemma 20. If $s \in(1,3 / 2], \varepsilon \in(0,1]$, and $\left(1-\partial_{x}^{2}\right) u_{0}$ does not change sign, then for any $T>0,\left\{u_{\varepsilon}\right\} \subset L^{\infty}\left([0, T] ; H^{q^{\prime}}\right)$, $\left\{\partial_{x} u_{\varepsilon}\right\} \subset L^{\infty}\left([0, T] ; H^{q^{\prime}-1}\right)$ with $q^{\prime} \in(1, s]$, and $\left\{\partial_{t} u_{\varepsilon}\right\} \subset$ $L^{\infty}\left([0, T] ; H^{r}\right)$ with $r \in(0, s-1]$.

Proof. By using (40), Lemma 19, and the Gronwall's inequality, we obtain that

$$
\begin{aligned}
\left\|u_{\varepsilon}\right\|_{H^{q^{\prime}}} \lesssim & \left\|J_{\varepsilon} u_{0}\right\|_{H^{q^{\prime}}} \\
& \quad \times \exp \left(C_{s} \int_{0}^{t}\left(\left\|\partial_{x} u_{\varepsilon}\right\|_{L^{\infty}}+\left\|\partial_{x} u_{\varepsilon}\right\|_{L^{\infty}}^{2}\right) d \tau\right) \\
\lesssim & \left\|u_{0}\right\|_{H^{s}} e^{C(T)} .
\end{aligned}
$$

Therefore $\left\|\partial_{x} u_{\varepsilon}\right\|_{H^{q^{\prime}-1}} \lesssim\left\|u_{0}\right\|_{H^{s}} e^{C(T)}$, where $C(T)$ only depends on T. Similarly, by using (62) and Lemma 19 we have

$$
\left\|\partial_{t} u_{\varepsilon}\right\|_{H^{r}} \lesssim\left\|u_{\varepsilon}\right\|_{H^{s}} \lesssim\left\|u_{0}\right\|_{H^{s}} e^{C(T)} .
$$

Thus for $q^{\prime} \in(1, s]$, we know $\left\{u_{\varepsilon}\right\}$ is uniformly bounded in $L^{\infty}\left([0, T] ; H^{q^{\prime}}\right)$, and $\left\{\partial_{x} u_{\varepsilon}\right\}$ is uniformly bounded in $L^{\infty}\left([0, T] ; H^{q^{\prime}-1}\right)$. Besides, $\left\{\partial_{t} u_{\varepsilon}\right\}$ is uniformly bounded in $L^{\infty}\left([0, T] ; H^{r}\right)$ with $r \in(0, s-1]$.

4.1. Existence of Global Weak Solution. Now we prove the existence of a global weak solution to problem (16).

Proposition 21. Suppose $u_{0} \in H^{s}(\mathbb{R})$ with $s \in(1,3 / 2]$ such that $\left(1-\partial_{x}^{2}\right) u_{0}$ does not change sign. Then (1) and (2) have a global weak solution $u \in L_{\mathrm{loc}}^{\infty}\left([0, \infty) ; H^{s}\right)$ in the sense of distribution. Moreover, $u_{x} \in L_{\mathrm{loc}}^{\infty}([0, \infty) \times \mathbb{T})$.

Proof. For an arbitrary fixed $T>0$, from Lemma 19, $\left\{u_{\varepsilon_{k}}\right\}$ and $\left\{\partial_{x} u_{\varepsilon_{k}}\right\}$ are bounded in $L^{\infty}([0, T] \times \mathbb{T})$. From Lemma 20, when $q^{\prime} \in(1, s]$ and $r \in(0, s-1]$, we have

$$
\begin{gathered}
\left\{u_{\varepsilon}\right\} \subset L^{2}\left([0, T] ; H^{q^{\prime}}\right), \\
\left\{\partial_{x} u_{\varepsilon}\right\} \subset L^{2}\left([0, T] ; H^{q^{\prime}-1}\right), \\
\left\{\partial_{t} u_{\varepsilon}\right\} \subset L^{2}\left([0, T] ; H^{r}\right) .
\end{gathered}
$$

Let $q^{\prime}=s, r=s-1$. By the Aubin compactness theorem [45], there is a subsequence $\left\{u_{\varepsilon_{k}}\right\} \subset\left\{u_{\varepsilon}\right\}$ such that $u_{\varepsilon_{k}}$ converges to some $u$ weakly in $L^{2}\left([0, T] ; H^{s}\right)$ and $\partial_{t} u_{\varepsilon_{k}} \rightarrow \partial_{t} u, \partial_{x} u_{\varepsilon_{k}} \rightarrow$ $\partial_{x} u$ weakly in $L^{2}\left([0, T] ; H^{s-1}\right)$ respectively. Moreover,

$$
\begin{gathered}
u_{\varepsilon_{k}} \longrightarrow u \quad \text { in } L^{2}\left([0, T] ; H^{q}\right) \text { strongly for } q \in(0, s), \\
\partial_{t} u_{\varepsilon_{k}} \longrightarrow \partial_{t} u, \quad \partial_{x} u_{\varepsilon_{k}} \longrightarrow \partial_{x} u \quad \text { in } L^{2}\left([0, T] ; H^{r}\right)
\end{gathered}
$$
strongly for $r \in(0, s-1)$. 
Thus, the sequences $\left\{\left(u_{\varepsilon_{k}}\right)^{n+1}\right\},\left\{\left(u_{\varepsilon_{k}}\right)^{n-1}\left(\partial_{x} u_{\varepsilon_{k}}\right)^{2}\right\},\left\{\left(u_{\varepsilon_{k}}\right)^{n-2}\right.$ $\left.\left(\partial_{x} u_{\varepsilon_{k}}\right)^{3}\right\}$ converge to $u^{n+1}, u^{n-1} u_{x}^{2}, u^{n-2} u_{x}^{3}$ strongly in $L^{2}\left([0, T] ; H^{r}\right)$ for any $r \in(0, s-1)$, respectively. Therefore, $u \in$ $C\left([0, T] ; H^{r}\right)$ is a solution to (16) in the sense of distribution. By Lemmas 19 and 20,u $\in L_{\mathrm{loc}}^{\infty}\left([0, \infty) ; H^{s}\right)$ and $u_{x} \in$ $L_{\text {loc }}^{\infty}([0, \infty) \times \mathbb{T})$.

\subsection{Uniqueness of the Global Weak Solution}

Proposition 22. Suppose $u_{0} \in H^{s}$ with $s \in(1,3 / 2]$. If $(1-$ $\left.\partial_{x}^{2}\right) u_{0}$ does not change sign, then the weak solution to (1) and (2) is unique.

Proof. Let $u, w$ be two solutions to (16) with the same initial data $u_{0}$; then $v=u-w$ satisfies

$$
v_{t}=-u^{n} v_{x}-f v \partial_{x} w-(F(u)-F(w)), \quad v(0, x)=0,
$$

where $f=\sum_{i=0}^{n-1}(w)^{n-1-i}(u)^{i}$. Calculating the $L^{2}$ energy of $v$ yields the equation

$$
\begin{aligned}
\frac{1}{2} \frac{d}{d t}\|v(t)\|_{L^{2}}^{2}= & -\int_{\mathbb{T}}(u)^{n} v_{x} \cdot v d x \\
& -\int_{\mathbb{T}} f v \partial_{x} w \cdot v d x-\int_{\mathbb{T}}(F(u)-F(w)) \cdot v d x .
\end{aligned}
$$

Since $\left(1-\partial_{x}^{2}\right) u_{0}$ does not change sign, Lemma 19 implies that $\left\|\partial_{x} u\right\|_{L^{\infty}} \leq\|u\|_{L^{\infty}} \lesssim\left\|u_{0}\right\|_{H^{1}}$ and $\left\|\partial_{x} w\right\|_{L^{\infty}} \leq\|w\|_{L^{\infty}} \lesssim\left\|u_{0}\right\|_{H^{1}}$. Employing the Cauchy-Schwarz inequality and integrating by parts yield

$$
\begin{gathered}
\int_{\mathbb{T}} u^{n} v_{x} \cdot v d x \leq\left\|u^{n-1} \partial_{x} u\right\|_{L^{\infty}}\|v\|_{L^{2}}^{2} \lesssim\|v\|_{L^{2}}^{2}, \\
\int_{\mathbb{T}} f v \partial_{x} w \cdot v d x \leq\|f\|_{L^{\infty}}\left\|\partial_{x} w\right\|_{L^{\infty}}\|v\|_{L^{2}}^{2} \lesssim\|v\|_{L^{2}}^{2} .
\end{gathered}
$$

For the estimate of the $L^{2}$ norm of $F\left(u_{\varepsilon}\right)-F\left(w_{\varepsilon}\right)$, we have

$$
\begin{aligned}
& \left\|F_{1}(u)-F_{1}(w)\right\|_{L^{2}} \\
& \leq\left\|u^{n+1}-w^{n+1}\right\|_{H^{-1}} \\
& \quad \leq\left\|\sum_{i=0}^{n} u^{n-i} w^{i}\right\|_{L^{\infty}}\|v\|_{L^{2}} \leq\|v\|_{L^{2}}, \\
& \left\|F_{2}(u)-F_{2}(w)\right\|_{L^{2}} \\
& \quad \leq\left\|u^{n-1}\left(u_{x}^{2}-w_{x}^{2}\right)\right\|_{H^{-1}}+\left\|w_{x}^{2}\left(u^{n-1}-w^{n-1}\right)\right\|_{H^{-1}} \\
& \quad \leq\left(\left\|u^{n-1}\left(u_{x}+w_{x}\right)\right\|_{L^{\infty}}+\left\|\sum_{i=0}^{n-2} u^{n-2-i} w^{i}\right\|_{L^{\infty}}\left\|w_{x}^{2}\right\|_{L^{\infty}}\right)\|v\|_{L^{2}} \\
& \quad \leq\|v\|_{L^{2}},
\end{aligned}
$$

$$
\begin{aligned}
& \left\|F_{3}(u)-F_{3}(w)\right\|_{L^{2}} \\
& \quad \leq\left\|u^{n-2}\left(u_{x}^{3}-w_{x}^{3}\right)\right\|_{H^{-2}}+\left\|w_{x}^{3}\left(u^{n-2}-w^{n-2}\right)\right\|_{H^{-2}} \\
& \quad \leq\left(\left\|u^{n-2} \cdot P\right\|_{L^{\infty}}+\left\|\sum_{i=0}^{n-3} u^{n-3-i} w^{i}\right\|_{L^{\infty}}\left\|w_{x}^{3}\right\|_{L^{\infty}}\right)\|v\|_{L^{2}} \\
& \quad \leq\|v\|_{L^{2}},
\end{aligned}
$$

where $P=u_{x}^{2}+u_{x} w_{x}+w_{x}^{2}$. Hence

$$
\int_{\mathbb{T}}(F(u)-F(w)) \cdot v d x \leqslant\|v\|_{L^{2}}^{2} .
$$

Combining these inequalities, we obtain that

$$
\frac{1}{2} \frac{d}{d t}\|v(t)\|_{L^{2}}^{2} \lesssim\|v\|_{L^{2}}^{2} .
$$

Using $v(0, x)=0$, we have $v=0$, which implies $u=w$.

Proof of Theorem 4. With the aid of Propositions 21 and 22, we complete the proof of Theorem 4.

\section{Ill-Posedness}

In this section we establish the ill-posedness of (1) and (2) in the sense that the solution map is not uniformly continuous from $H^{s}$ into $C\left([0, T] ; H^{s}\right)$ with $s<3 / 2$ either. Firstly, we show that (1) and (2) possess periodic peaked solutions.

\subsection{Existence of Periodic Peaked Solutions}

Proposition 23. Let $c>0$ and $\theta=x-c t-2 \pi[(x-c t) / 2 \pi]-\pi$. Then $u(t, x)=\gamma \cosh \theta$ is a periodic peaked solution to (1) if and only if $\gamma=c^{1 / n} \operatorname{sech} \pi$. Moreover, $u(t, \cdot) \in H^{s}$ with $s<3 / 2$.

Proof. Let $H_{1}=\partial_{t}\left(1-\partial_{x}^{2}\right) u, H_{2}=(1 /(n+1)) \partial_{x}(1-$ $\left.\partial_{x}^{2}\right)\left(u^{n+1}\right), H_{3}=\partial_{x}\left(u^{n+1}+((2 n-1) / 2) u^{n-1} u_{x}^{2}\right)$, and $H_{4}=$ $((n-1) / 2) u^{n-2} u_{x}^{3}$. Then (1) can be expressed as $\sum_{i=1}^{4} H_{i}=0$. Assuming that $u(x, t)=\gamma \cosh \theta$ is the periodic solution to (1), we have

$$
\begin{aligned}
u_{x} & =\gamma \sinh \theta, \\
u_{x x} & =\gamma \cosh \theta-2 \gamma \sinh \pi \delta_{c t},
\end{aligned}
$$

where $\delta_{c t}$ is the periodic Dirac delta function at $x=c t(\bmod$ $2 \pi)$. Thus

$$
H_{1}=-2 c \gamma \sinh \pi \delta_{c t}^{\prime} .
$$

Direct computation shows that

$$
\begin{aligned}
\left(u^{n+1}\right)_{x x}= & (n+1) \gamma^{n+1} \\
\times & \left(\cosh ^{n+1}(\theta)+n \cosh ^{n-1}(\theta) \sinh ^{2}(\theta)\right. \\
& \left.\quad-2 \cosh ^{n}(\pi) \sinh \pi \delta_{c t}^{\prime}\right)
\end{aligned}
$$




$$
\begin{aligned}
&=(n+1) \gamma^{n+1} \\
& \times\left((n+1) \cosh ^{n+1}(\theta)-n \cosh ^{n-1}(\theta)\right. \\
&\left.\quad-2 \cosh ^{n}(\pi) \sinh \pi \delta_{c t}^{\prime}\right) .
\end{aligned}
$$

Therefore, we have

$$
\begin{aligned}
H_{2}= & \frac{1}{n+1} \partial_{x}\left(u^{n+1}-\left(u^{n+1}\right)_{x x}\right) \\
= & \gamma^{n+1}\left(\left(-n^{2}-2 n\right) \cosh ^{n}(\theta) \sinh \theta\right. \\
& \left.\quad+\left(n^{2}-n\right) \cosh ^{n-2}(\theta) \sinh \theta\right) \\
& +2 \gamma^{n+1} \cosh ^{n-1}(\pi) \sinh \pi \delta_{c t}^{\prime} .
\end{aligned}
$$

Using (80), we can compute that

$$
\begin{aligned}
H_{3}=\gamma^{n+1}( & 3 n \cosh ^{n}(\theta) \sinh \theta \\
& \left.+\frac{(n-1)(2 n-1)}{2} \cosh ^{n-2}(\theta) \sinh ^{3}(\theta)\right) \\
=\gamma^{n+1}( & \frac{2 n^{2}+3 n-1}{2} \cosh ^{n}(\theta) \sinh \theta \\
& \left.\quad-\frac{(n-1)(2 n-1)}{2} \cosh ^{n-2}(\theta) \sinh \theta\right) .
\end{aligned}
$$

Similarly,

$$
\begin{aligned}
H_{4} & =\frac{n-1}{2} \gamma^{n+1} \cosh ^{n-2}(\theta) \sinh ^{3}(\theta) \\
& =\frac{n-1}{2} \gamma^{n+1}\left(\cosh ^{n}(\theta) \sinh \theta-\cosh ^{n-2}(\theta) \sinh \theta\right) .
\end{aligned}
$$

Putting these results together, we see that

$$
\sum_{i=1}^{4} H_{i}=2 \gamma^{n+1} \cosh ^{n}(\pi) \sinh \pi \delta_{c t}^{\prime}-2 c \gamma \sinh \pi \delta_{c t}^{\prime}=0,
$$

which implies $\gamma=c^{1 / n} \operatorname{sech} \pi$.

Obviously, if $u(t, x)=c^{1 / n} \operatorname{sech} \pi \cosh (x-c t-2 \pi[(x-$ $c t) / 2 \pi]-\pi)$, we have

$$
\begin{aligned}
\widehat{u}(t, \xi)= & c^{1 / n} \operatorname{sech} \pi \\
& \times \int_{\mathbb{T}} e^{-i x \xi} \cosh \left(x-c t-2 \pi\left[\frac{x-c t}{2 \pi}\right]-\pi\right) d x \\
= & 2 c^{1 / n} \operatorname{sech} \pi \cdot e^{-i c t \xi} \\
& \times \int_{0}^{2 \pi}\left(e^{(1-i \xi) x-\pi}+e^{-(1+i \xi) x+\pi}\right) d x \\
= & 2 c^{1 / n} \tanh \pi \cdot \frac{e^{-i c t \xi}}{1+\xi^{2}} .
\end{aligned}
$$

Therefore, when $s<3 / 2$, we have

$$
\begin{aligned}
\|u\|_{H^{s}}^{2} & =\sum_{\xi=-\infty}^{+\infty}\left(1+|\xi|^{2}\right)^{s}|\widehat{u}|^{2} \\
& =4 \tanh ^{2}(\pi) c^{2 / n} \sum_{\xi=\infty}^{+\infty}\left(1+|\xi|^{2}\right)^{s-2}<\infty .
\end{aligned}
$$

Hence $u(t, x)=c^{1 / n} \operatorname{sech} \pi \cosh \theta \in H^{s}$ with $s<3 / 2$.

5.2. Proof of Theorem 5. By Proposition 23, we know that (1) has two sequences of periodic peakon (weak) solutions:

$$
\begin{aligned}
& u_{m}(t, x)=a_{m}^{1 / n} \operatorname{sech} \pi \cosh \left(x-a_{m} t-2 \pi\left[\frac{x-a_{m} t}{2 \pi}\right]-\pi\right), \\
& v_{m}(t, x)=c_{m}^{1 / n} \operatorname{sech} \pi \cosh \left(x-c_{m} t-2 \pi\left[\frac{x-c_{m} t}{2 \pi}\right]-\pi\right),
\end{aligned}
$$

where $a_{m}, c_{m} \in \mathbb{R}$ are constants velocity which will be specified later. By (88), we know

$$
\begin{aligned}
& \widehat{u_{m}}(t, \xi)=2 a_{m}^{1 / n} \tanh (\pi) \cdot \frac{e^{-i a_{m} t \xi}}{1+\xi^{2}} \\
& \widehat{v_{m}}(t, \xi)=2 c_{m}^{1 / n} \tanh (\pi) \cdot \frac{e^{-i c_{m} t \xi}}{1+\xi^{2}} .
\end{aligned}
$$

Note $s<3 / 2$; when $t=0$, we have

$$
\begin{aligned}
& \left\|u_{m}(0, \cdot)-v_{m}(0, \cdot)\right\|_{H^{s}}^{2} \\
& =\sum_{\xi=-\infty}^{+\infty}\left(1+|\xi|^{2}\right)^{s}\left|\widehat{u_{m}}(0, \xi)-\widehat{v_{m}}(0, \xi)\right|^{2} \\
& =4 \tanh ^{2}(\pi)\left(a_{m}^{1 / n}-c_{m}^{1 / n}\right)^{2} \sum_{\xi=-\infty}^{+\infty}\left(1+|\xi|^{2}\right)^{s-2} \\
& =\left(C_{1}(s)\right)^{2}\left(a_{m}^{1 / n}-c_{m}^{1 / n}\right)^{2},
\end{aligned}
$$

where $C_{1}(s)=2 \tanh (\pi)\left(\sum_{\xi=-\infty}^{+\infty}\left(1+|\xi|^{2}\right)^{s-2}\right)^{1 / 2}<+\infty$. 
When $t>0$, it follows that

$$
\begin{aligned}
& \left\|u_{m}(t, \cdot)-v_{m}(t, \cdot)\right\|_{H^{s}}^{2} \\
& =\sum_{\xi=-\infty}^{+\infty}\left(1+|\xi|^{2}\right)^{s}\left|\widehat{u_{m}}(t, \xi)-\widehat{v_{m}}(t, \xi)\right|^{2} \\
& =4 \tanh ^{2}(\pi) \sum_{\xi=-\infty}^{+\infty}\left(1+|\xi|^{2}\right)^{s-2} \\
& \quad \times\left|a_{m}^{1 / n} e^{-i a_{m} t \xi}-c_{m}^{1 / n} e^{-i c_{m} t \xi}\right|^{2} \\
& =\left\|u_{m}(0, x)-v_{m}(0, x)\right\|_{H^{s}}^{2} \\
& \quad+8 \tanh ^{2}(\pi)\left(a_{m} c_{m}\right)^{1 / n} \sum_{\xi=-\infty}^{+\infty} \frac{1-\cos \left(\left(a_{m}-c_{m}\right) t \xi\right)}{\left(1+|\xi|^{2}\right)^{2-s}} \\
& \geq 8 \tanh ^{2}(\pi)\left(a_{m} c_{m}\right)^{1 / n} \sum_{\xi=-\infty}^{+\infty} \frac{1-\cos \left(\left(a_{m}-c_{m}\right) t \xi\right)}{\left(1+|\xi|^{2}\right)^{2-s}} .
\end{aligned}
$$

Note that $\left(1-\cos \left(\left(a_{m}-c_{m}\right) t \xi\right)\right) /\left(1+|\xi|^{2}\right)^{2-s} \geq 0$. For any fixed $t>0$, we choose $m_{0} \in \mathbb{N}$ large enough such that $\left[m_{0} \pi / 3 t\right]>1$ and take $c_{m}=m^{2 n(1+|s|)}, a_{m}=c_{m}+1 / m, m \geq m_{0}$. Let $\xi_{1}=$ $[m \pi / 2 t+1], \xi_{2}=[3 m \pi / 2 t] ;$ then we have $\xi_{2}-\xi_{1} \geq m \pi / 3 t>0$ and

$$
\begin{aligned}
8 \tanh ^{2}(\pi)\left(a_{m} c_{m}\right)^{1 / n} & \times \sum_{\xi=-\infty}^{+\infty} \frac{1-\cos \left(\left(a_{m}-c_{m}\right) t \xi\right)}{\left(1+|\xi|^{2}\right)^{2-s}} \\
\geq & 8 \tanh ^{2}(\pi)\left(a_{m} c_{m}\right)^{1 / n} \\
& \times \sum_{\xi=\xi_{1}}^{\xi_{2}} \frac{1-\cos \left(\left(a_{m}-c_{m}\right) t \xi\right)}{\left(1+|\xi|^{2}\right)^{2-s}} \\
\geq & 8 \tanh ^{2}(\pi) m^{4(1+|s|)} \\
& \times \sum_{\xi=[m \pi / 2 t+1]}^{[3 m \pi / 2 t]} \frac{1-\cos (t \xi / m)}{\left(1+|\xi|^{2}\right)^{2-s}} \\
\geq & 8 \tanh (\pi) m^{4(1+|s|)} \\
& \times \sum_{\xi=[m \pi / 2 t+1]}^{[3 m \pi / 2 t]} \frac{1}{\left(2|\xi|^{2}\right)^{2-s}} \\
\geq & \frac{2^{5-s} \tanh ^{2}(\pi)}{3^{5-2 s} \pi^{3-2 s}} t^{3-2 s} m^{2 s+4|s|+1} \\
= & \left(C_{2}(s)\right)^{2} t^{3-2 s} m^{2 s+4|s|+1},
\end{aligned}
$$

where $C_{2}(s)=\sqrt{2^{5-s} \tanh ^{2}(\pi) / 3^{5-2 s} \pi^{3-2 s}}$. Therefore, from (95), we have

$$
\left\|u_{m}(t, \cdot)-v_{m}(t, \cdot)\right\|_{H^{s}} \geq C_{2}(s) t^{3 / 2-s} m^{s+2|s|+1 / 2} .
$$

Since

$$
a_{m}-c_{m}=\left(a_{m}^{1 / n}-c_{m}^{1 / n}\right)\left(\sum_{j=0}^{n-1} a_{m}^{(n-1-j) / n} c_{m}^{j / n}\right),
$$

we have

$$
a_{m}^{1 / n}-c_{m}^{1 / n}=\frac{a_{m}-c_{m}}{\sum_{j=0}^{n-1} a_{m}^{(n-1-j) / n} c_{m}^{j / n}} \leq \frac{1 / m}{n c_{m}^{(n-1) / n}}<\frac{1}{m} .
$$

Hence we can infer from (93) that

$$
\begin{aligned}
& \left\|u_{m}(0, \cdot)-v_{m}(0, \cdot)\right\|_{H^{s}}^{2} \\
& \quad \leq \frac{4 \tanh ^{2}(\pi)}{m^{2}} \sum_{\xi=-\infty}^{+\infty}\left(1+|\xi|^{2}\right)^{s-2}=\frac{\left(C_{1}(s)\right)^{2}}{m^{2}} .
\end{aligned}
$$

Combining (97) and (100), we complete the proof of Theorem 5 .

Remark 24. From Theorems 2 and 5, we see $s=3 / 2$ is the critical index of regularity for well-posedness in Sobolev space $H^{s}$ for (1) and (2).

Remark 25. From the dynamical system point of view, when $s<3 / 2$, we can deduce that none of the periodic peakons is Lyapunov stable. In fact, for all $\varepsilon>0$, for any periodic peakon $u_{c}(t, x)$ with $c>0$, following (93) and (95), we see that, for $\eta>0$,

$$
\begin{aligned}
& \left\|u_{c+\eta}(0, x)-u_{c}(0, x)\right\|_{H^{s}}^{2} \\
& \leq\left(4 \tanh ^{2}(\pi) \sum_{\xi=-\infty}^{+\infty}\left(1+|\xi|^{2}\right)^{s-2}\right) \eta^{2}, \\
& \left\|u_{c+\eta}(t, x)-u_{c}(t, x)\right\|_{H^{s}}^{2} \\
& \quad \geq 8 \tanh ^{2}(\pi) c^{2 / n} \sum_{\xi=-\infty}^{+\infty} \frac{1-\cos (\eta t \xi)}{\left(1+|\xi|^{2}\right)^{2-s}} \\
& \geq \frac{2^{5-s} \tanh ^{2}(\pi)}{3^{5-2 s} \pi^{3-2 s}} t^{3-2 s} \eta^{3-2 s} c^{2 / n} .
\end{aligned}
$$

If we let $\eta=\eta(s, \varepsilon)>0$ and $t=t_{\eta}$ such that

$$
\eta^{2}<\frac{\varepsilon^{2}}{\left(4 \tanh ^{2}(\pi) \sum_{\xi=-\infty}^{+\infty}\left(1+|\xi|^{2}\right)^{s-2}\right)}, \quad t=t_{\eta}=\frac{\pi}{\eta},
$$

then we have

$$
\begin{gathered}
\left\|u_{c+\eta}(t, x)-u_{c}(t, x)\right\|_{H^{s}}^{2}<\varepsilon^{2}, \\
\left\|u_{c+\eta}\left(t_{\eta}, x\right)-u_{c}\left(t_{\eta}, x\right)\right\|_{H^{s}}^{2} \geq \frac{2^{5-s} \tanh ^{2}(\pi)}{3^{5-2 s}} c^{2 / n} .
\end{gathered}
$$

Hence $u_{c}$ is indeed unstable in the sense of Lyapunov. 


\section{Conflict of Interests}

The authors declare that there is no conflict of interests regarding the publication of this paper.

\section{Acknowledgment}

This work is supported by the National Natural Science Foundation of China (no. 11461014).

\section{References}

[1] A. S. Fokas and B. Fuchssteiner, "Symplectic structures, their Bäcklund transformations and hereditary symmetries," Physica $D$, vol. 4, no. 1, pp. 47-66, 1981.

[2] R. Camassa and D. D. Holm, "An integrable shallow water equation with peaked solitons," Physical Review Letters, vol. 71, no. 11, pp. 1661-1664, 1993.

[3] A. Constantin and J. Escher, "Wave breaking for nonlinear nonlocal shallow water equations," Acta Mathematica, vol. 181, no. 2, pp. 229-243, 1998.

[4] C. E. Kenig, G. Ponce, and L. Vega, "Well-posedness and scattering results for the generalized Korteweg-de Vries equation via the contraction principle," Communications on Pure and Applied Mathematics, vol. 46, no. 4, pp. 527-620, 1993.

[5] G. B. Whitham, Linear and Nonlinear Waves, John Wiley \& Sons, New York, NY, USA, 1980.

[6] A. Constantin, "The trajectories of particles in Stokes waves," Inventiones Mathematicae, vol. 166, no. 3, pp. 523-535, 2006.

[7] A. Constantin and J. Escher, "Particle trajectories in solitary water waves," American Mathematical Society, vol. 44, no. 3, pp. 423-431, 2007.

[8] A. Constantin and J. Escher, "Analyticity of periodic traveling free surface water waves with vorticity," Annals of Mathematics, vol. 173, no. 1, pp. 559-568, 2011.

[9] A. Constantin, "On the Cauchy problem for the periodic Camassa-Holm equation," Journal of Differential Equations, vol. 141, no. 2, pp. 218-235, 1997.

[10] G. Misiołek, "Classical solutions of the periodic Camassa-Holm equation," Geometric and Functional Analysis, vol. 12, no. 5, pp. 1080-1104, 2002.

[11] A. Constantin, "Existence of permanent and breaking waves for a shallow water equation: a geometric approach," Annales de l'Institut Fourier, vol. 50, no. 2, pp. 321-362, 2000.

[12] A. Constantin and J. Escher, "Global existence and blow-up for a shallow water equation," Annali della Scuola Normale Superiore di Pisa. Classe di Scienze, vol. 26, no. 2, pp. 303-328, 1998.

[13] A. Constantin and J. Escher, "Well-posedness, global existence, and blowup phenomena for a periodic quasi-linear hyperbolic equation," Communications on Pure and Applied Mathematics, vol. 51, no. 5, pp. 475-504, 1998.

[14] H. P. McKean, "Breakdown of a shallow water equation," The Asian Journal of Mathematics, vol. 2, no. 4, pp. 867-874, 1998.

[15] Z. Jiang, L. Ni, and Y. Zhou, "Wave breaking of the CamassaHolm equation," Journal of Nonlinear Science, vol. 22, no. 2, pp. 235-245, 2012.

[16] Z. Xin and P. Zhang, "On the weak solutions to a shallow water equation," Communications on Pure and Applied Mathematics, vol. 53, no. 11, pp. 1411-1433, 2000.
[17] A. Bressan and A. Constantin, "Global conservative solutions of the Camassa-Holm equation," Archive for Rational Mechanics and Analysis, vol. 183, no. 2, pp. 215-239, 2007.

[18] A. Bressan and A. Constantin, "Global dissipative solutions of the Camassa-Holm equation," Analysis and Applications, vol. 5, no. 1, pp. 1-27, 2007.

[19] H. Holden and X. Raynaud, "Global conservative solutions of the Camassa-Holm equation-a Lagrangian point of view," Communications in Partial Differential Equations, vol. 32, no. 10-12, pp. 1511-1549, 2007.

[20] H. Holden and X. Raynaud, "Dissipative solutions for the Camassa-Holm equation," Discrete and Continuous Dynamical Systems. Series A, vol. 24, no. 4, pp. 1047-1112, 2009.

[21] Z. Jiang, Y. Zhou, and M. Zhu, "Large time behavior for the support of momentum density of the Camassa-Holm equation," Journal of Mathematical Physics, vol. 54, no. 8, Article ID 081503, 2013.

[22] A. A. Himonas, G. Misiołek, G. Ponce, and Y. Zhou, "Persistence properties and unique continuation of solutions of the Camassa-Holm equation," Communications in Mathematical Physics, vol. 271, no. 2, pp. 511-522, 2007.

[23] V. Novikov, "Generalizations of the Camassa-Holm equation," Journal of Physics. A. Mathematical and Theoretical, vol. 42, no. 34, 342002, 14 pages, 2009.

[24] X. Geng and B. Xue, "An extension of integrable peakon equations with cubic nonlinearity," Nonlinearity, vol. 22, no. 8, pp. 1847-1856, 2009.

[25] A. N. Hone and J. P. Wang, "Integrable peakon equations with cubic nonlinearity," Journal of Physics A: Mathematical and Theoretical, vol. 41, no. 37, Article ID 372002, 2008.

[26] A. N. Hone, H. Lundmark, and J. Szmigielski, "Explicit multipeakon solutions of Novikov's cubically nonlinear integrable Camassa-Holm type equation," Dynamics of Partial Differential Equations, vol. 6, no. 3, pp. 253-289, 2009.

[27] L. Ni and Y. Zhou, "Well-posedness and persistence properties for the Novikov equation," Journal of Differential Equations, vol. 250, no. 7, pp. 3002-3021, 2011.

[28] X. Wu and Z. Yin, "A note on the Cauchy problem of the Novikov equation," Applicable Analysis, vol. 92, no. 6, pp. 11161137, 2013.

[29] X. Wu and Z. Yin, "Global weak solutions for the Novikov equation," Journal of Physics A: Mathematical and Theoretical, vol. 44, no. 5, Article ID 055202, 17 pages, 2011.

[30] Z. Jiang and L. Ni, "Blow-up phenomenon for the integrable Novikov equation," Journal of Mathematical Analysis and Applications, vol. 385, no. 1, pp. 551-558, 2012.

[31] F. Tiğlay, “The periodic Cauchy problem for Novikov's equation," International Mathematics Research Notices, vol. 2011, no. 20, pp. 4633-4648, 2011.

[32] A. A. Himonas and C. Holliman, "The Cauchy problem for the Novikov equation," Nonlinearity, vol. 25, no. 2, pp. 449-479, 2012.

[33] K. Grayshan, "Peakon solutions of the Novikov equation and properties of the data-to-solution map," Journal of Mathematical Analysis and Applications, vol. 397, no. 2, pp. 515-521, 2013.

[34] H. Tang, Y. Zhao, and Z. Liu, "A note on the solution map for the periodic Camassa-Holm equation," Applicable Analysis, vol. 93, no. 8, pp. 1745-1760, 2014.

[35] H. Tang and Z. Liu, "Continuous properties of the solution map for the Euler equations," Journal of Mathematical Physics, vol. 55, no. 3, Article ID 031504, 2014. 
[36] H. Tang, S. Shi, and Z. Liu, "The dependences on initial data for the b-family equation in critical Besov space," Monatshefte für Mathematik, 2014.

[37] H. Tang and Z. Liu, "The Cauchy problem for a two-component Novikov equation in the critical Besov space," Journal of Mathematical Analysis and Applications, vol. 423, no. 1, pp. 120135, 2015.

[38] A. A. Himonas and C. Holliman, "The Cauchy problem for a generalized CAMassa-Holm equation," Advances in Differential Equations, vol. 19, no. 1-2, pp. 161-200, 2014.

[39] A. Himonas and G. Misiołek, "The Cauchy problem for an integrable shallow-water equation," Differential and Integral Equations, vol. 14, no. 7, pp. 821-831, 2001.

[40] T. Kato and G. Ponce, "Commutator estimates and the Euler and Navier-Stokes equations," Communications on Pure and Applied Mathematics, vol. 41, no. 7, pp. 891-907, 1988.

[41] M. Taylor, Partial Differential Equations III: Nonlinear Equations, Springer, New York, NY, USA, 1996.

[42] A. A. Himonas and C. Kenig, "Non-uniform dependence on initial data for the $\mathrm{CH}$ equation on the line," Differential and Integral Equations, vol. 22, no. 3-4, pp. 201-224, 2009.

[43] M. Taylor, "Commutator estimates," Proceedings of the American Mathematical Society, vol. 131, no. 5, pp. 1501-1507, 2003.

[44] S. Alinhac, Blowup for Nonlinear Hyperbolic Equations, vol. 17 of Progress in Nonlinear Differential Equations and Their Applications, Birkhäuser, Boston, Mass, USA, 1995.

[45] J. Lions, Quelques Méthodes de Résolution des Problèmes aux Limites Nonlinéaires, Dunod, Gauthier-Villars, Paris, France, 1969. 


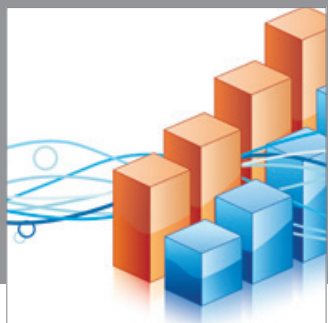

Advances in

Operations Research

mansans

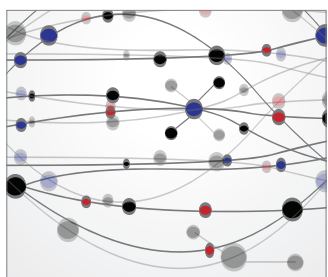

The Scientific World Journal
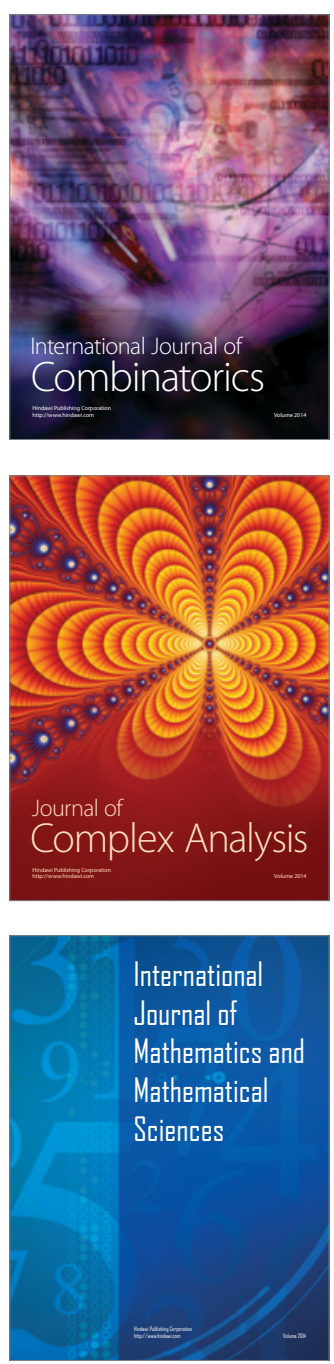
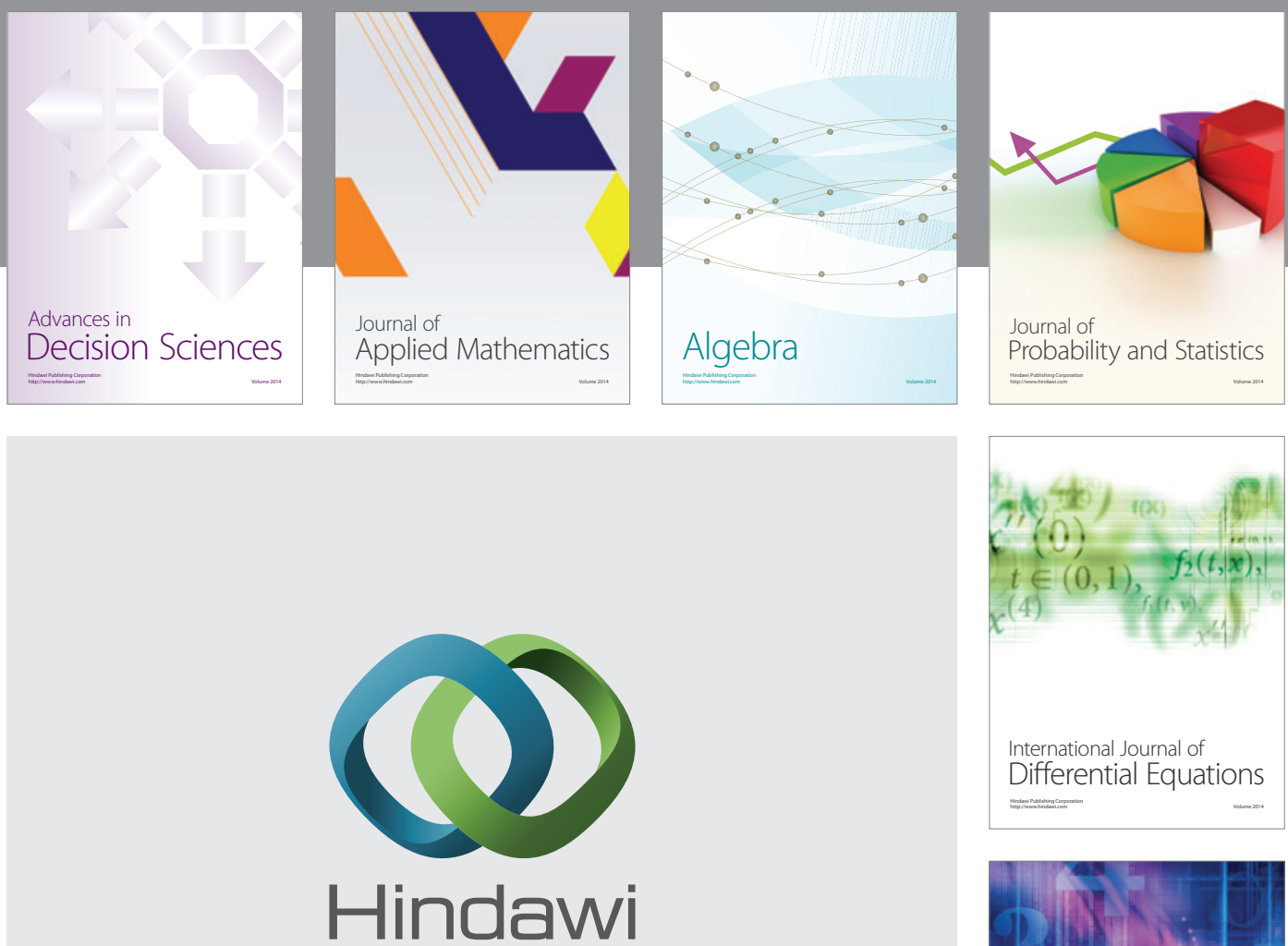

Submit your manuscripts at http://www.hindawi.com
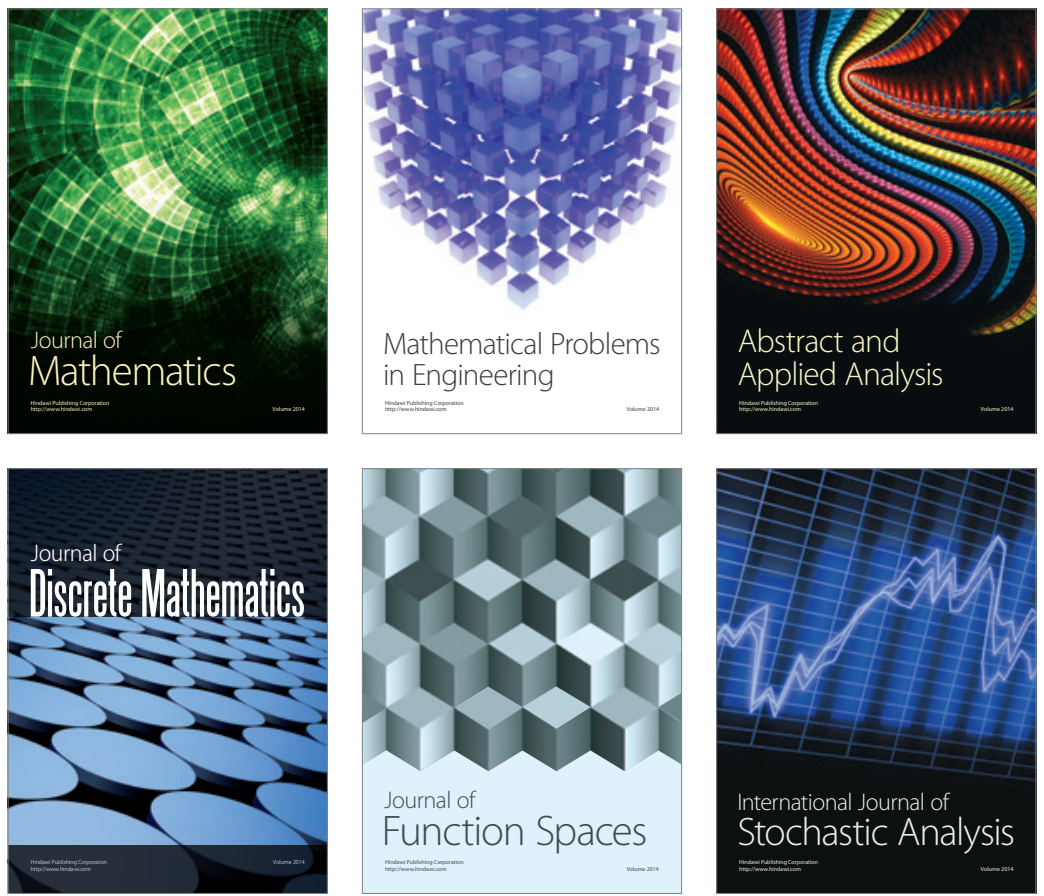

Journal of

Function Spaces

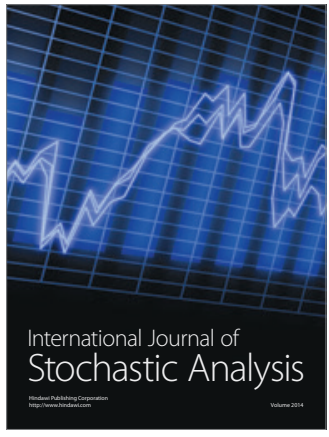

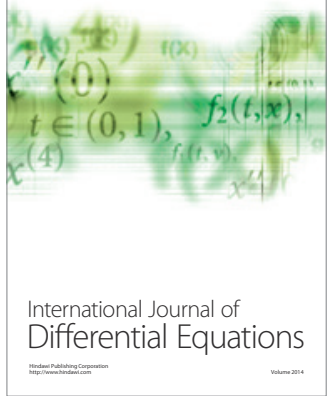
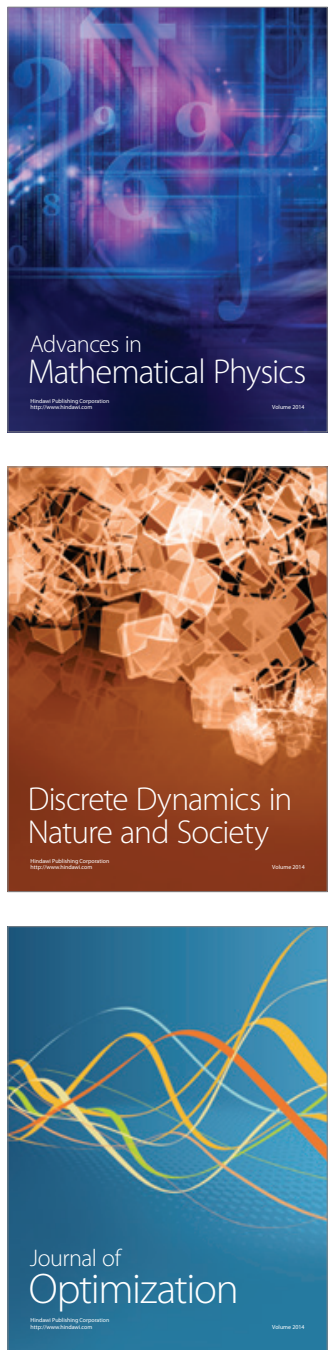